\title{
BMJ Open Barriers and facilitators to medication adherence: a qualitative study with general practitioners
}

\author{
Kirsi Kvarnström, ${ }^{1}$ Marja Airaksinen, ${ }^{1}$ Helena Liira ${ }^{2,3}$
}

\begin{abstract}
To cite: Kvarnström K, Airaksinen M, Liira $\mathrm{H}$. Barriers and facilitators to medication adherence: a qualitative study with general practitioners. BMJ Open 2018;8:e015332. doi:10.1136/ bmjopen-2016-015332
\end{abstract}

Received 27 November 2016 Revised 9 November 2017 Accepted 28 November 2017

D) Check for updates

${ }^{1}$ Clinical Pharmacy Group, Division of Pharmacology and Pharmacotherapy, Faculty of Pharmacy, University of Helsinki, Finland

${ }^{2}$ General Practice, School of Medicine, University of Western Australia, Crawley, Western Australia, Australia

${ }^{3}$ Department of General Practice and Primary Health Care,

Helsinki University Central

Hospital, University of Helsinki, Finland

Correspondence to Kirsi Kvarnström;

kirsi.kvarnstrom@helsinki.fi

\begin{abstract}
Background General practitioners (GPs) manage the drug therapies of people with chronic diseases, and poor adherence to medication remains a major challenge. Objective This qualitative study examined GPs' insights into non-adherence and ways of overcoming this problem. Methods We ran four focus groups comprising $16 \mathrm{GPs}$ at the Kirkkonummi Health Centre (Southern Finland). Interviews were audiotaped, transcribed verbatim and analysed by inductive content analysis.

Main results The two main themes in the discussions with the GPs were non-adherence in the care of chronic disease and increased need for medicine information. The medication management challenges identified were related to: patient-specific factors, the healthcare system, characteristics of drug therapies and the function and role of healthcare professionals as a team. To improve the situation, the GPs offered a number of solutions: improved coordination of care, better patient education and IT systems as well as enhanced interprofessional involvement in the follow-up of patients.

Discussion and conclusions With an ageing population, the GPs were increasingly confronted with non-adherence in the care of chronic diseases. They had mostly a positive attitude towards organising care in a more interprofessional manner. To support medication adherence and self-management, the GPs appreciated pharmacists' assistance especially with patients with polypharmacy and chronic diseases.
\end{abstract}

\section{INTRODUCTION}

Globally, the prevalence of chronic disease is increasing, yet adherence to treatment regimens remains persistently low. In developed countries, only about $50 \%$ of all patients treat their chronic disease according to the instructions. ${ }^{2}$ Non-adherence is associated with poorer clinical outcomes, and ageing populations in particular experience negative effects of poor adherence. ${ }^{34}$ Multiple diseases and polypharmacy among older adults further challenge the good coordination of care.

Non-adherence appears to be a difficult problem to resolve and remains a global concern for healthcare. ${ }^{5}$ A Cochrane review included almost 200 randomised controlled trials (RCTs), and it was evident that in only

\section{Strengths and limitations of this study}

The strength of this study was a comprehensive sampling of the general practitioners (GPs) of all the three healthcare centres in Kirkkonummi and the doctors interviewed included both the experienced and the newcomers.

- The interviews were thoroughly analysed to define the two overarching themes and the subthemes, and recruitment was continued until the saturation of the data.

- The limitation of this focus group study was that the GPs were recruited from only one medium large health centre in Finland.

- The 16 GPs out of a total of 25 in the health centre voluntarily participated in four focus groups and therefore they might be more interested in interprofessional collaboration and medication adherence than the other nine GPs.

a minority of the lowest risk of bias, RCTs improved both adherence and clinical outcomes. ${ }^{6}$ Despite the fact that intervention studies in optimal circumstances may show some results, their effectiveness is even more limited in real-life clinical settings. The difficulties in measuring the adherence further complicate the matter. Healthcare professionals also tend to overestimate patients' adherence in routine clinical practice. ${ }^{7}$

The Cochrane review concluded that one explanation for non-effectiveness is the lack of a thorough understanding of the adherence problems. ${ }^{6}$ Overall, non-adherence is a complex process and more understanding is needed from the qualitative research perspective. So far, qualitative studies have focused on the viewpoint of patients, ${ }^{8}$ healthcare professionals ${ }^{910}$ and the interactions between different professionals. ${ }^{11}$ Type of interaction, communication and trust appear to be among the essential themes in these studies. ${ }^{911}$

The Cochrane review advised that shifting medication counselling to allied healthcare providers seems a reasonable and potentially cost-effective strategy. ${ }^{6}$ Although there 
is no clear evidence that interprofessional interventions are more effective than single-handed ones, they appear to offer the best opportunity to improve clinical outcomes. ${ }^{12}$ Patients do not self-manage alone and they would benefit from different types of support by different healthcare professionals. ${ }^{13}$

General practitioners (GPs) are responsible for much of the medication prescribing and counselling for chronic diseases. The aim of this study was to explore GPs' insights into medication adherence and to assess the perceived barriers, facilitators and ideas for improving patients' adherence in routine clinical practice. Our study sought to broaden understanding of the primary care doctors' struggle against the multifaceted phenomenon of non-adherence. We also aimed to study GPs' attitudes towards having pharmacists as team members in primary care and the need for pharmacists' involvement in patient education and counselling services.

\section{METHODS}

\section{Study design and data collection}

The study was conducted in the Kirkkonummi Health Centre, which is a municipal health service for the 36000 inhabitants of Kirkkonummi, Finland. Kirkkonummi health centre is a single centre administratively but operating in three venues: the main health centre in Kirkkonummi centre and two smaller regional health centres in the Kirkkonummi area (in Masala and in Veikkola). We invited GPs working in the health centre to participate and ran focus groups in all of the three regional areas of service between October 2010 and May 2011.

The qualitative design was chosen in order to gain a deeper understanding of GPs' perceptions towards medication adherence and the problems GPs thought patients might have experienced in following instructions for their medical care. In this study, we used a topic guide of semistructured questions to allow the GPs to approach the subject from a personal point of view (box 1). Two convenors ran the focus groups: a moderator (KK) and

\section{Box 1 Topic guide for the focus group discussions}

All questions were open ended and aimed at encouraging
discussions
In your opinion, how active are your patients in managing
their chronic conditions? How do they succeed with their self-
management?
How do you coach their self-management?
If it were possible to refer some patients to a pharmacist for a
consultation, what type of patients would you refer?
Which are those groups of patients that have the largest problems
with self-management? Do they have something in common?
How do you help patients to commit to their treatments? What are
the challenges?
How do you find the inter-professional collaboration in the
coordination of care?

a facilitator (either HL or another local GP). The main researcher had a background in pharmacy, while the other facilitators were GPs. The interviewees knew the backgrounds of the researchers.

We used purposive sampling to select GPs in our study. The GPs participating in the focus groups were recruited by email invitation. In the recruitment process, age, gender, experience and work place were taken into account. Recruitment was continued until the saturation of the data. Not all invited doctors participated in the study because of workload or absence from work. The interviews were audiotaped and transcribed verbatim.

The focus group interviews were analysed by conventional inductive content analysis. ${ }^{14}$ First, meaningful units of the transcripts were manually coded. Next categories were derived from these units and finally condensed during the analysis. KK did the initial coding and analysis. All authors read the transcripts and results and the conclusions were confirmed by discussion and consultation.

The study was conducted in accordance with good scientific practice. Based on Finnish ethical principles of research, there was no need to apply for permission from the hospital district's ethics committee for this voluntary focus group interview study. ${ }^{15}$ All participants gave their written informed consent.

\section{RESULTS}

Four focus groups, with a total of 16 GPs, were convened and the characteristics of the participants are presented in table 1 . The interviews lasted from 60 to $90 \mathrm{~min}$. Two of them took place in the main health centre of Kirkkonummi, one was conducted in Veikkola and another in Masala, the two regional health centres of the municipality. Both young and more experienced GPs were represented in the interviews (table 1).

Table 1 Characteristics of the general practitioners interviewed in the focus groups

\begin{tabular}{lc}
\hline Variable & (n) \\
\hline Gender & \\
\hline Female & 12 \\
\hline Male & 4 \\
\hline Age & \\
\hline $25-30$ & 4 \\
\hline $31-40$ & 4 \\
\hline $41-54$ & 6 \\
\hline 255 & 2 \\
\hline Education & \\
\hline Licensed medical doctor & 5 \\
\hline Medical doctor in the specialist training of general & 4 \\
practice & 7 \\
\hline Specialist in general practice & \\
\hline
\end{tabular}


Box 2 Barriers to good medication adherence according to the general practitioners (GPS)

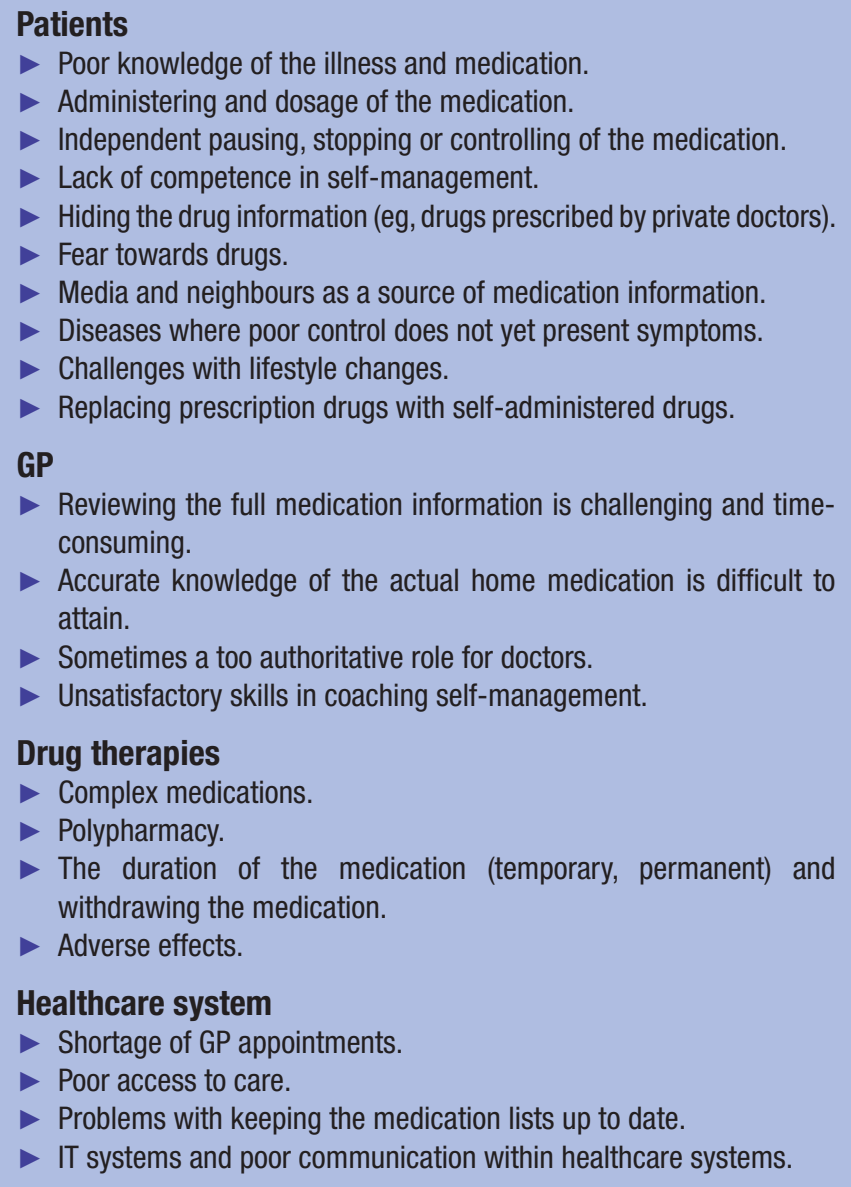

\section{Poor adherence in medication management}

The first of the main themes that emerged during the coding process was poor adherence, which continues to remain a challenge in care. According to the GPs, the main barriers to good adherence were related to patient-specific factors, the role of the doctor and the health system. Box 2 presents a detailed list of the collected themes of the perceived barriers.

\section{Patient-related factors of self-management}

According to the focus group discussions, the GPs were quite burdened with non-adherence to medication. The patients managed their chronic disease poorly and it was easy for the GPs to agree that probably at least half of their patients experienced difficulties with their medication adherence. The GPs also noticed that problems accumulated in certain patient groups.

Those patients who have difficulty have problems in differing ways. That's the key thing in my opinion. If you decide to focus on just one problem at the consultation, you would still need to have a plan for the rest of them. You cannot ignore the other problems.

According to the GPs, there was a huge variation in the self-management in general as well as in medication management among different patient groups. A few treated their disease exactly as ordered using their own Excel tables to document the outcome of the care. Others did not attend GP's appointments or manage their disease as agreed. The GPs thought that these patients were confused with their medication and often did not know how the medicines should be taken.

Many patients can't distinguish many of their medications at all. And when you ask, they say: "that white little tablet...'

In all four focus group discussions, the GPs wanted to talk about their especially problematic patients, who had many diseases and multiple medications. Examples of special risk groups mentioned by the GPs are presented in table 2. Also, in the case of home care clients and their caregivers, the adherence was considered even more challenging, mostly because of lack of time for home care.

The challenge often was with the instructions. When a new drug was commenced, the GP explained the medication to the patient. On the other hand, the patients often decided for themselves how they should take their medication, and this was not always in accordance with the GP's instruction.

I've observed that the patients with warfarin do not dare to tell they've taken anti-inflammatory drugs when nothing else helps. Then we have great trouble in adjusting the warfarin dosage and do not know the real reason.

Patients did not necessarily understand why the medication had been prescribed for them. Patients might regard the GP's questions on adherence as interference even though the purpose was to clarify the issues and to find the best alternative to treat their disease. The GPs knew the patients made independent decisions on their treatment and they hoped those decisions would not be detrimental.

\section{The role of the doctor}

According to the GPs, the authoritative role taken by doctors may demotivate patients from taking responsibility for their medication management. The doctors were aware that patients did not always tell them the truth about their use of medicines.

Patients regard doctors as an authority and they try to please us. They sometimes try to embellish things with their drug use.

However, the GPs had other ways to observe the real use of drugs.

Often you see it from the prescriptions that they renew that there is the equivalent of 200 tablets not yet purchased and the patient says they have taken them every day...

You see it from the outcomes of care as well, most evidently with cholesterol drugs. 
Table 2 Examples of especially challenging patient groups with regard to medication adherence according to the GPs

\section{Challenging patient groups Citations}

Patients with hypercholesterolaemia I cannot see why there is not more discussion of simvastatin. I'm in a true simvastatin rumba with my patients...(refers to media attention)

Patients with asthma

Many patients with asthma take their medication in a totally irrational manner. For example, if they have symptoms and they have to walk the dog, they may take first the treatment drug and then the opening drug.

They so easily stop taking the drug if they are in good balance.

\begin{tabular}{|c|c|}
\hline Patients with diabetes & Diabetes and hypertension are the difficult ones as they do not give symptoms. \\
\hline Patients with hypertension & $\begin{array}{l}\text { I have had those hypertension patients who only take the drugs when they feel a little } \\
\text { dizzy. }\end{array}$ \\
\hline Caregivers & $\begin{array}{l}\text { I've met caregivers who do not have information of the appropriate care. If they are not } \\
\text { present when the medication is prescribed, they may not understand it and be able to } \\
\text { assist in the medication management. }\end{array}$ \\
\hline
\end{tabular}

The GPs' opinion was that they sometimes lacked the skills in supporting patients when it came to guidance on day-to-day issues and counselling at home.

There is also the fact that I don't feel so confident in there... I feel the nurses are better at guiding patients on the practical issues of what to do at home.

\section{The health system}

The GPs thought that the length of consultations and the amount of work expected of them often did not match. The GPs regarded supporting self-management as a difficult job because they did not have enough time to go through all the necessary details.

The patients' consultations with different doctors made it difficult to keep the medication lists up to date because of the various IT systems in primary and secondary care and, in addition, in private healthcare. The GPs only saw the medication lists of the health records in the public health system. The medication changes made by private doctors and sometimes also those made by specialists were not easily accessible. However, the patients were not aware of this and did not understand that they should tell the doctor of all medications they were taking and how they were administering them.

The challenge is that just as you think you have succeeded to collect a thorough list of all medications, you notice the next time that there is something else as well; and it is quite annoying.

I have learnt that specific questions are important. You need to ask about things such as glaucoma or postmenopausal symptoms to find out whether private specialists have prescribed glaucoma medication or hormonal replacement to them.

\section{Better medication information for the patient}

The second major theme in the interviews was the increased need for medication information as a solution for poor adherence. According to the GPs, the risk groups particularly needed more medication counselling. The focus groups provided a list of proposed solutions, which are presented in box 3. Many of them were related to better interprofessional cooperation.

\section{More patient empowerment}

Although the GPs generally agreed about taking charge of medications, they wished the patients would take more responsibility for their own care. The GPs did not oppose changing their role from an authoritative one to one more like that of a coach.

...the idea would be that you are there like a coach in a way and that the patients would take the responsibility for their medication themselves.

According to the GPs' experience, the instructions should be repeated because the patients easily forgot the information they received. The GPs also acknowledged that they needed better communication strategies.

The style of the guidance matters, so that the patient can absorb the information. It needs to be tailored to their needs. Some of them understand immediately, for others you really need to repeat and simplify things.

You must be respectful and careful in my opinion. On the other hand I do press for my point of view.

Peer support was seen as a strengthening factor for patients' medication adherence. However, the doctors emphasised that there should be a healthcare professional to facilitate the group discussion. 
Box 3 Facilitators to better medication adherence according to the GPS

Patient
Focus on health outcomes of self-management and drug therapies.
Support for patients to better understand their disease and its
management.
Pharmacists as coaches for drug therapies.
Medication counselling for caregivers.
Peer groups for chronic conditions and training in peer groups.
Doctor
Interprofessional practices for updating the drug lists before yearly
check-ups.
Continuity of care and permanent doctor-patient relationships.
Equal relationships with patients, with a coaching attitude.
Setting achievable goals.
Drug therapies
Interprofessional interventions: medication reconciliation (nurse or
pharmacist), medicines optimisation (pharmacist) and medication
review (pharmacist).
Combination of products to minimise the number of medicines.
Checking and teaching the right use of medical devices.
Healthcare system
Interprofessional practices.
Interprofessional interventions: medication reconciliation, medicines
optimisation and medication review.

The opportunity to discuss with peers is important. However, there must be a professional included so that the discussions don't start to go in the wrong direction.

\section{Continuity of care}

It takes time to build trustworthy relationships with patients. According to the GPs, a long-lasting GP-patient relationship was seen as a strengthening factor for medicine adherence. If the GP can, together with the patient, set achievable goals and follow how the patients achieve the goals, the patient will be committed to the goals and motivated to achieve them.

I believe a steady doctor-patient relationship improves medication adherence. There is no such adherence if the patient zigzags between various doctors.

It is good to have common goals, goals that the patient is committed to and that we have agreed on.

\section{More interprofessional practices}

According to the GPs, one way to tackle the non-adherence would be with more interprofessional support for the patient. This would allow for more support and depth for the medication management.

With the challenging ones there are better and worse periods of motivation. If there was another professional to say the right words at the right moment...
The GPs' appreciated help from other members of the interprofessional teams especially with complex patients and medications. There was a need for more collaboration between GPs and the other healthcare professionals to tackle the problems of polypharmacy. Interactions and adverse effects weaken medication adherence and self-management and are an obstacle to achieving the desired outcome.

Sometimes it's really hard to figure out the medication. In those cases it would be useful to have other team members to sort out the actual medication and explain things.

The doctors would appreciate nurses' help with annual appointments for chronic diseases. If the nurse interviewed the patient before the GP's appointment and the medication list was updated before the patient saw the GP, the GPs would have all the necessary information to make decisions.

\section{Process improvement in healthcare systems}

Also on the healthcare level, better interprofessional processes would help the doctors to collaborate with the other healthcare professionals. According to the GPs, it would benefit all healthcare because 'no one person would need to do everything'.

Sometimes the lack of indication for a medication was not questioned at GP appointments because it was prescribed in secondary care. In cases like this, drugs prescribed originally for acute reasons may have become regular medications.

Sometimes we are too humble with regard to secondary care. If they have prescribed a drug, we take it as granted. We should also critically appraise those drugs. The secondary care may not have had the true information of the current medication of the patient.

According to the GPs, the patients sent home from secondary care would need more medication counselling and guidance with the self-management of their medication. Especially, those patients with serious cardiovascular events were often confused about the situation. They were often discharged from hospital quickly and without a proper understanding of their situation.

The patient had chest pain and ended up in hospital and had balloon angioplasty, and the next thing he noticed was they gave him prescriptions and sent him home saying: 'See your GP'. It is sometimes difficult for these patients to figure out the need for all these new medications.

\section{The role of the pharmacist}

According to the GPs, medication reconciliation and rationalisation of medicine use were among the tasks for which doctors would appreciate help from pharmacists. 
For those patients with a magnitude of drugs, it may be such a chaotic process. Drugs prescribed from many sources, and a total lack of overall planning.

If a pharmacist figured out the actual medication the patient uses at home and the medication list was updated before the GPs appointment, this would be embraced by GPs.

There is so much to deal with during the yearly check-ups and finding out about medication easily takes all the time. Without that there would be more time for other things, discussion and planning with the patient.

I agree we'd need help with the multi-morbidity and multi-prescribed. Patients sometimes try themselves to reduce the drugs and would certainly appreciate advice.

The GPs agreed the medication reviews made by the pharmacist, for patients with multiple medications or new symptoms, would support them in decision-making and help patients to cope with the medication.

I thought of those patients who would like to reduce the number of their drugs. This is one situation where a pharmacist's review might be useful.

...Especially those with polypharmacy and also the home-dwelling older people who start to have problems coping. They cannot figure out the need for a review themselves.

...Reducing anticholinergic drugs and interactions sometimes help them to cope at home enormously.

Sometimes the GPs experienced challenges evaluating if the symptom was an adverse effect, interaction or a new illness. In those cases, the medication review made by the pharmacist might help them in decision-making. It might also be a solution in cases where the patient's status has deteriorated due to an unclear reason.

...With problematic symptoms, sometimes you wonder is this due to medication interactions or something new going on.

Having a pharmacist as an inseparable part of the interprofessional care team was welcomed by the GPs. They thought it would offer better opportunities for the rational use of medicines and support patients' medication adherence.

I believe colleagues would welcome help like this. Sorting out the long medication lists takes so much of our time.

\section{DISCUSSION}

\section{Main findings}

The GPs described poor medication adherence as one of the largest problems with which they were faced. ${ }^{16}$ With an ageing population they had to tackle increasingly complex medication management issues. In addition, there were large individual differences in patients' needs and skills, which further complicated the guiding of patients' self-management. An increased need for medication information was the key issue for the GPs, and it was thought that better interprofessional care and better IT systems would ameliorate medication management.

The GPs had observed that their authoritative role hindered patients from mentioning the problems with their medication. Patients often tried to please the doctors and did not necessarily tell their doctors whether they had taken their medication correctly. A recent study from France observed a large discordance between patient and physician evaluations of drug adherence and drug importance, ${ }^{17}$ which may be due to a lack of patient-centred communication.

Another challenge was the lack of a method to measure patients' self-management. ${ }^{18}$ Doctors often did not have tools to resolve patients' adherence to medication, which led to unclear situations. The GPs explained that they tried to analyse patients' adherence with methods such as observing the frequency of prescription renewals. However, it is not a reliable indicator of the adherence and gives only references about the adherence. The method of clinical outcomes also does not reflect patients' medication adherence and doctors in fact tend to overestimate their adherence. ${ }^{7}$

Other challenges with medication adherence included the lack of information and incompatible IT solutions. It was difficult for the GPs to keep the medication lists up-to-date because of the various IT systems in primary and secondary care and, in addition, in private healthcare. The GPs wished for shared IT systems, to which everyone involved in care has an access, to better handle multiple medications. There are high hopes for better IT and electronic decision support among health professionals and such systems may also enhance their interprofessional collaboration. ${ }^{19}$

A shortage of time during the GPs' appointments did not help the situation either. ${ }^{5}$ Other factors that hampered medication adherence included patient-related factors; patients were often discouraged from taking the medication by the information in the package leaflet or by a friend's opposing advice. A qualitative study from the UK observed that the vast amount of side effects and drug interactions commonly described in package leaflets sometimes caused feelings of fear and anxiety in patients. ${ }^{8}$

On the other hand, the GPs thought that there were positive trends taking place, including the change seen in their role whereby they were acting more as the patients' coach or guide instead of an authority figure. When patients are more involved in their own medication management, it could strengthen their medication adherence and make them more like an active participant. ${ }^{20}$

Ideally, the patient should be the focus of all interventions and an active member of the care team. Also, GPs felt strongly that an open and long-lasting doctor-patient 
relationship increases patients' adherence to medication. It allows patients' difficulties to be understood under day-to-day conditions.

The GPs strongly favoured better interprofessional collaboration to accompany the medical information given by GPs in order to empower the patient. The interprofessional cooperation can help to increase adherence and self-management and eliminate patients' suspicions about the medication. ${ }^{11}$ When the message to the patient is the same regardless of which healthcare professional counsels the patient about medication, it increases trust and produces a favourable attitude towards the desired outcome.

Based on this study, the GPs were not opposed to having pharmacists in the interprofessional care team to solve problems in polypharmacy. Medication reconciliation and medication review are interventions performed by pharmacists, which could provide the GPs with decision support for medication management and it could also enable the safe and effective use of medicines with better outcomes. ${ }^{21}$ Collaboration between GPs and pharmacists continues to be an area of interest, and a questionnaire has been developed to measure GPs' attitudes towards collaborating with pharmacists. ${ }^{22}$

\section{Limitations and strengths}

The limitation of this focus group study was that the GPs were recruited from only one medium large health centre. The 16 GPs out of a total of 25 in the centre voluntarily participated in four focus groups, and therefore they might be more interested in interprofessional collaboration and medication adherence than the other nine GPs. Because of the small sample, the generalisability of the findings is limited. However, we believe our findings contribute to an increased understanding of the complexity of medication adherence.

The strength of this study was a comprehensive sampling of the GPs of all the three healthcare centres in Kirkkonummi. The doctors interviewed included both the experienced and the newcomers. The interviews were thoroughly analysed to define the two overarching themes and the subthemes. Recruitment was continued until saturation of the data and until any new information was obtained.

This qualitative study in primary care was aimed at understanding the GPs' everyday challenges with self-management. The GPs' insight into barriers and facilitators to facilitate medication adherence and their solutions to the problem might advise researchers who plan further patient-centred interventions in order to support adherence in primary care.

\section{CONCLUSIONS}

Those GPs interviewed were concerned with poor medication adherence, which they considered the major problem in guiding the self-management of people with chronic disease. To overcome the problems, they suggested better interprofessional cooperation and more open communication with patients. They wished to work closer with nurses and pharmacists to help people with chronic disease. The GPs also emphasised the need for better IT systems to keep medication information up to date and also that more focus should be given to advising patients on drugs.

Acknowledgements We would like to thank Kaija Hannula for her help in conducting the focus groups. We are grateful to all the doctors who participated in this study and would like to thank them for their involvement and effort.

Contributors Two convenors ran the focus groups: a moderator (KK) and a facilitator (either HL or another local general practitioner, Kaija Hannula). KK did the initial coding and analysis. All authors read the transcripts and results and the conclusions were chosen by discussion and consultation. All authors participated in the critical revision of the manuscript.

Funding This research received no specific grant from any funding agency in the public, commercial or not-for-profit sectors.

Competing interests None declared.

Patient consent Not required.

Ethics approval The board of the Kirkkonummi Health Centre, which was the local ethics committee, granted the study permission. Based on Finnish ethical principles of research there was no need to apply for permission from the hospital district's ethics committee for this voluntary focus group interview study.

Provenance and peer review Not commissioned; externally peer reviewed.

Data sharing statement All requests should be directed to the corresponding author.

Open Access This is an Open Access article distributed in accordance with the Creative Commons Attribution Non Commercial (CC BY-NC 4.0) license, which permits others to distribute, remix, adapt, build upon this work non-commercially, and license their derivative works on different terms, provided the original work is properly cited and the use is non-commercial. See: http://creativecommons.org/ licenses/by-nc/4.0/

(c) Article author(s) (or their employer(s) unless otherwise stated in the text of the article) 2018. All rights reserved. No commercial use is permitted unless otherwise expressly granted.

\section{REFERENCES}

1. Brown MT, Bussell JK. Medication adherence: WHO cares? Mayo Clin Proc 2011;86:304-14.

2. Naderi SH, Bestwick JP, Wald DS. Adherence to drugs that prevent cardiovascular disease: meta-analysis on 376,162 patients. Am J Med 2012;125:882-7.

3. Mukhtar O, Weinman J, Jackson SH. Intentional non-adherence to medications by older adults. Drugs Aging 2014;31:149-57.

4. Costa E, Giardini A, Savin M, et al. Interventional tools to improve medication adherence: review of literature. Patient Prefer Adherence 2015;9:1303.

5. Clyne W, Mshelia C, McLachlan S, et al. A multinational crosssectional survey of the management of patient medication adherence by European healthcare professionals. BMJ Open 2016;6:e009610.

6. Nieuwlaat R, Wilczynski N, Navarro T, et al. Interventions for enhancing medication adherence. Cochrane Database Syst Rev 2014;11:CD000011.

7. Kekäle M, Talvensaari K, Koskenvesa P, et al. Chronic myeloid leukemia patients' adherence to peroral tyrosine kinase inhibitors compared with adherence as estimated by their physicians. Patient Prefer Adherence 2014;8:1619-27.

8. Herber OR, Gies V, Schwappach D, et al. Patient information leaflets: informing or frightening? A focus group study exploring patients' emotional reactions and subsequent behavior towards package leaflets of commonly prescribed medications in family practices. BMC Fam Pract 2014;15:1.

9. Gale NK, Greenfield S, Gill P, et al. Patient and general practitioner attitudes to taking medication to prevent cardiovascular disease after receiving detailed information on risks and benefits of treatment: a qualitative study. BMC Fam Pract 2011;12:1. 
10. Jamison J, Graffy J, Mullis R, et al. Stroke survivors', caregivers' and GPs' attitudes towards a polypill for the secondary prevention of stroke: a qualitative interview study. BMJ Open 2016;6:e010458.

11. Rathbone AP, Mansoor SM, Krass I, et al. Qualitative study to conceptualise a model of interprofessional collaboration between pharmacists and general practitioners to support patients' adherence to medication. BMJ Open 2016;6:e010488.

12. Mansoor SM, Krass I, Aslani P. Multiprofessional interventions to improve patient adherence to cardiovascular medications. $J$ Cardiovasc Pharmacol Ther 2013;18:19-30.

13. Dwarswaard J, Bakker EJ, van Staa A, et al. Self-management support from the perspective of patients with a chronic condition: a thematic synthesis of qualitative studies. Health Expect 2016;19:194-208.

14. Silverman D. Doing qualitative research: a practical handbook. 4th Edn. London: SAGE Publications Ltd, 2013.

15. National Advisory Board on Research Ethics. Ethical principles of research in the humanities and social and behavioural sciences and proposals for ethical review. 2009. www.tenk.fi/en/tenk-guidelines

16. World Health Organization. Adherence to long-term therapies: evidence for action. WHO: Switzerland, Geneva, 2003.
17. Sidorkiewicz S, Tran VT, Cousyn C, et al. Discordance between drug adherence as reported by patients and drug importance as assessed by physicians. Ann Fam Med 2016;14:415-21.

18. Lehmann A, Aslani P, Ahmed R, et al. Assessing medication adherence: options to consider. Int J Clin Pharm 2014;69:36-55.

19. Koskela T, Sandström S, Mäkinen J, et al. User perspectives on an electronic decision-support tool performing comprehensive medication reviews - a focus group study with physicians and nurses. BMC Med Inform Decis Mak 2016;16:6-16.

20. Menichetti J, Libreri C, Lozza E, et al. Giving patients a starring role in their own care: a bibliometric analysis of the on-going literature debate. Health Expect 2016;19:516-26.

21. Leikola S. Development and application of comprehensive medication review procedure to community-dwelling elderly: University of Helsinki, 2012. http://urn.fi/URN:ISBN:978-952-10-7698-5

22. Van C, Costa D, Mitchell B, et al. Development and validation of a measure and a model of general practitioner attitudes toward collaboration with pharmacists. Res Social Adm Pharm 2013;9:688-99. 